\title{
Conception d'activités pédagogiques en formation infirmière au travers de la pensée critique de Freire / Developing pedagogical activities for nursing education inspired by Freire's critical perspective
}

Jérôme Leclerc-Loiselle

Université de Montréal, jerome.leclerc-loiselle@umontreal.ca

Émilie Dufour

Université de Montréal, emilie.dufour.3@umontreal.ca

Jacinthe I. Pepin

Université de Montréal, jacinthe.pepin@umontreal.ca

Follow this and additional works at: https://qane-afi.casn.ca/journal

Part of the Social and Philosophical Foundations of Education Commons

\section{Recommended Citation}

Leclerc-Loiselle, Jérôme; Dufour, Émilie; and Pepin, Jacinthe I. (2019) "Conception d'activités pédagogiques en formation infirmière au travers de la pensée critique de Freire / Developing pedagogical activities for nursing education inspired by Freire's critical perspective," Quality Advancement in Nursing Education - Avancées en formation infirmière: Vol. 5: Iss. 2, Article 4.

DOI: https://doi.org/10.17483/2368-6669.1187

This Article is brought to you for free and open access by Quality Advancement in Nursing Education - Avancées en formation infirmière. It has been accepted for inclusion in Quality Advancement in Nursing Education - Avancées en formation infirmière by an authorized editor of Quality Advancement in Nursing Education - Avancées en formation infirmière. 


\section{Conception d'activités pédagogiques en formation infirmière au travers de la pensée critique de Freire / Developing pedagogical activities for nursing education inspired by Freire's critical perspective}

\section{Cover Page Footnote}

Les deux premiers auteurs (Leclerc-Loiselle et Dufour) ont une contribution à parts égales. Les auteurs tiennent à souligner que cet article a initialement été réfléchi dans le cadre d'un cours de doctorat sur la formation en sciences infirmières. 


\section{Introduction}

L'augmentation de la complexité des situations de soins, la place grandissante des technologies de l'information et de la communication, la pénurie d'infirmières et les changements démographiques ont largement transformé la pratique infirmière au cours des dernières années (Benner, Sutphen, Leonard et Day, 2010; Ironside, 2001; Rich et Nugent, 2010). Benner et al. (2010), dans leurs travaux sur la formation infirmière, en appelaient à une transformation radicale de la formation afin de répondre aux changements sociétaux et à ceux du système de santé. Ces travaux, datant de près de 10 ans, soutenaient alors que les méthodes d'apprentissage entretiennent encore trop souvent un savoir décontextualisé, dissocient le milieu clinique du milieu académique et intègrent peu de réflexions critiques sur l'action clinique. Frenk et al. (2010), dans leurs travaux s'inscrivant dans la commission internationale sur la formation des professionnels de la santé du $\mathrm{XXI}^{\mathrm{e}}$ siècle, en arrivaient à un constat similaire et proposaient une redéfinition du cursus et des modalités de formation pour l'ensemble des professionnels de la santé. Malgré la parution du rapport il y a près d'une décennie, un développement conceptuel des programmes de formation professionnelle est toujours nécessaire pour répondre à la complexité du milieu de la santé (Feller, 2018).

Le rapport de Frenk et al. (2010) situe les programmes de formation professionnelle actuels dans une réforme nommée « apprentissage par problèmes » qui propose une approche centrée sur l'étudiant. Cette approche fait suite à une première réforme basée principalement sur les travaux de Flexner (1910), qui soutenaient l'intégration d'une approche scientifique à la formation professionnelle.

Bien que la seconde réforme ait contribué à rapprocher la formation des milieux cliniques et communautaires, ces auteurs suggèrent que cette réforme est insuffisante pour répondre à la complexité des enjeux sanitaires mondiaux et aux besoins de contextualisation des compétences développées. Ils soutiennent que la réforme « apprentissage par compétences basé sur une vision systémique » pourrait permettre de répondre davantage aux enjeux actuels de la formation professionnelle. L'apprentissage de type transformationnel, basé sur le développement d'un raisonnement critique chez l'étudiant, constitue une composante centrale de cette seconde réforme (Frenk et al., 2010).

En sciences infirmières, des méthodes d'apprentissage par problèmes issues de la seconde réforme rapportée par Frenk et al. (2010) sont à ce jour encore très présentes dans les universités canadiennes (Goudreau et al., 2009; Rosenau, Watson, Vye-Rogers et Dobbs, 2015). La troisième réforme proposée par Frenk et al. (2010), qui s'articule autour de l'apprentissage transformationnel, est en émergence dans les réflexions sur la recherche en formation infirmière (Pepin et al., 2017). Cependant, afin de poursuivre l'intégration de ce type d'apprentissage dans la formation infirmière, les programmes universitaires doivent avoir recours à des repères théoriques explicites et cohérents (McAllister, 2011). Pour ce faire, Frenk et al. (2010) suggèrent que les travaux de Freire $(1970,1973,1998)$, de par la place centrale qu'y occupe la notion de réflexion critique, peuvent s'avérer pertinents.

Nous considérons ainsi qu'une réflexion s'impose sur les repères philosophiques qui sous-tendent la formation infirmière. Par l'intégration de la réflexion critique guidant une action informée, par la contextualisation des connaissances et par des interactions horizontales entre enseignant et apprenant, certains repères conceptuels issus de la pensée de Freire offrent des pistes de solutions envisageables en réponse aux critiques formulées par Frenk et al. (2010) et 
Benner et al. (2010) sur la formation professionnelle actuelle. Si certains écrits se sont penchés sur l'apport des travaux de Freire en sciences infirmières (Bond Butts et Saucier Lundy, 2003; Forneris et Peden-McAlpine, 2006), aucun article identifié ne fait état du développement d'activités pédagogiques de niveau universitaire de premier cycle fondé sur cette pensée.

Cet article vise ainsi à proposer une mise en action du processus d'apprentissage transformationnel dans le développement d'activités pédagogiques en sciences infirmières au travers de la pensée de Freire. À la suite d'une description théorique de la pensée de ce philosophe de l'éducation, nous prendrons en exemples les activités pédagogiques d'un cours d'intégration de l'approche salutogénique à la pratique infirmière d'un programme de formation universitaire de premier cycle en sciences infirmières au Canada. Une description sommaire des activités pédagogiques du cours, conceptuellement soutenue par les travaux de Freire, sera présentée. Cette proposition d'activités se veut non dogmatique. Les mêmes notions théoriques issues de la pensée de Freire auraient pu mener au développement d'autres activités pédagogiques tout aussi valides. De plus, il est à noter que l'approche salutogénique est ici utilisée en exemple dans les processus pédagogiques du cours présenté. Ainsi, cette proposition pourrait s'appliquer à d'autres contenus cohérents avec les repères philosophiques et issus de différents cours de la formation infirmière. Nous espérons que cette description incitera les concepteurs de cours et de programmes de formation infirmière à porter attention aux prémisses conceptuelles qui guident leurs activités pédagogiques ainsi qu'à considérer l'apprentissage transformationnel, selon un ou plusieurs penseurs, comme perspective pour préparer les futurs professionnels ${ }^{1}$ à des soins de qualité dans un contexte de complexité.

\section{Repères conceptuels issus des travaux de Freire}

Paolo Freire est un pédagogue et philosophe brésilien. Jusqu'à sa mort en 1997, il fut un important critique et réformateur des pratiques pédagogiques qu'il qualifie de «bancaires », soit des pratiques où l'apprenant est conçu comme un objet dénué de savoirs. Inscrit dans un contexte dictatorial de servitude populaire, Freire (1970) développe une conception de l'éducation dite libératrice. Cette approche pédagogique est caractérisée par un processus d'apprentissage inquisitif et vise à outiller l'individu de façon à questionner les connaissances et les valeurs produites et transmises dans la société. Freire (1973) conçoit la réalité comme étant construite historiquement et politiquement. La réalité du monde est différente en fonction des contextes dans lesquels les individus évoluent, mais commune dans ce qu'il nomme la lutte pour la libération (Freire, 1973). Cette libération de l'individu consiste en un processus qui lui permet d'accéder à un pouvoir d'action et de transformation du monde. Freire (1973) conçoit essentiellement qu'une telle libération s'effectue au travers d'une conscientisation. La conscientisation, comme objectif de la formation, se définit comme un processus social dans lequel les sujets actifs approfondissent la conscience qu'ils ont, tant de la réalité socioculturelle qui structure leur vie, que de leur capacité de transformer cette réalité (Freire, 1970). À partir de cette conception de la conscientisation, nous retenons pour cet article trois notions centrales aux travaux de Freire $(1970,1973,1998)$ qui peuvent servir de repères au développement d'activités pédagogiques en sciences infirmières, soit les interactions, la contextualisation et la réflexion critique.

\footnotetext{
${ }^{1}$ Le masculin est utilisé dans ce texte dans le seul but de faciliter la lecture; il inclut les personnes de tout genre sans discrimination.
} 
En s'appuyant sur les travaux de Freire (1970, 1998), nous réfléchissons d'abord l'apprentissage comme s'effectuant au travers d'interactions, tant entre les étudiants et les formateurs (professeurs et autres groupes enseignants), qu'entre les étudiants eux-mêmes. Cette notion d'interactions sous-tend un partage de connaissances entre le formateur et l'apprenant, plutôt qu'un transfert vertical des connaissances, permettant ainsi un échange des représentations de chacun (Freire, 1998). Freire (1970) soutient qu'une conception de rôles partagés où tous les acteurs peuvent se positionner à la fois comme éducateurs et comme apprenants permet une participation active nécessaire à la conscientisation des sujets sur les rapports qu'ils entretiennent avec le monde social. Bien qu'il distingue les espaces respectifs occupés par l'éducateur et l'apprenant, Freire (1998) soutient qu'ils peuvent mutuellement contribuer à enrichir leurs apprentissages. Considérant le caractère inévitablement normatif de l'éducation, Freire (1998) conçoit comme essentiel pour l'éducateur de reconnaître ses propres idées et positions de façon à permettre un espace de dialogue empreint d'ouverture.

Nous concevons ensuite que la notion de contextualisation des connaissances est nécessaire à l'apprentissage de l'étudiant. Pour Freire (1970, 1973), cette notion de contextualisation se rapporte à un ancrage des notions théoriques dans une réalité qui tient compte d'un ensemble de dimensions tant sociales, culturelles ou politiques. La contextualisation favorise l'apprentissage en facilitant les représentations de l'étudiant en contexte de pratique (Freire, 1970, 1973). Pour ce faire, Freire (1970) soutient qu'une curiosité épistémique, qui se traduit par une capacité à lier la théorie et la pratique à partir des connaissances acquises ou développées antérieurement, est fondamentale à l'apprentissage. Un engagement de l'étudiant dans l'intégration des concepts théoriques est nécessaire à l'apprentissage, au même titre que l'est une application pratique (Freire, 1970). L'apprentissage se traduit donc en partie par la capacité de l'étudiant à transformer en connaissances ses expériences vécues et à mobiliser les connaissances acquises pour développer de nouveaux savoirs (Freire, 1970). Ainsi, les activités pédagogiques devraient permettre une mobilisation contextualisée des expériences antérieures de l'étudiant.

Nous concevons finalement la place centrale qu'occupe la réflexion critique dans le processus d'apprentissage. Pour Freire (1970, 1998), il s'agit d'un processus central à la conscientisation de l'individu, qui lui permet de poser des actions de façon consciente et raisonnée, en comprenant les conséquences de ses actions. Selon cette perspective, les activités pédagogiques devraient offrir un espace de réflexion à l'étudiant, tant sur soi que sur le monde social dans lequel il évolue, de même que fournir les outils nécessaires à l'intégration de la réflexion critique dans la poursuite de ses apprentissages. Dans une perspective freirienne, la réflexion critique est centrale à la problématisation, qui constitue une stratégie pédagogique dans laquelle l'étudiant développe des connaissances en questionnant et en analysant de manière active et critique une réalité donnée (Freire, 1970). La réflexion critique est ainsi liée à la praxis, qui dans une vision récursive permet des interactions constantes entre la théorie et son application pratique au travers d'une réflexion critique permettant d'ajuster, de modifier ou de transformer les actions (Freire, 1970). Parmi les auteurs en sciences infirmières qui promeuvent la réflexion critique et le questionnement du statu quo socio-culturel et politique, Chinn et Kramer (2015) identifient la praxis comme le processus par lequel le savoir émancipatoire peut être développé. Ces auteurs situent le mode émancipatoire, qui appelle à l'action pour réduire les inégalités et les injustices sociales, au centre et en relation avec l'ensemble des savoirs mobilisés. Ainsi, la réflexion critique comme mécanisme central à l'apprentissage permettrait de 
questionner l'ensemble des savoirs de façon à en modifier leur application pratique en fonction du contexte social.

\section{Utilisation des notions de Freire en formation en sciences infirmières}

Les notions issues des travaux de Freire ont été utilisées de façon ponctuelle en sciences infirmières pour réfléchir différentes dimensions de la formation dans tous les cycles d'études universitaires. Bond Butts et Saucier Lundy (2003) suggèrent que les repères philosophiques, en agissant comme un ancrage, sont centraux à l'évolution du niveau de réflexion des étudiants doctoraux et, ultimement, à l'avancement des sciences infirmières. Bond Butts et Saucier Lundy (2003) ont ainsi eu recours aux travaux de Freire (1998) pour soutenir une réflexion critique nécessaire à l'introduction de perspectives philosophiques nouvelles à travers l'utilisation de repères connus et significatifs pour les étudiants. Bien que le propos présenté par ces auteurs offre une perspective concrète permettant de réfléchir la conception d'un programme de formation de futurs enseignants en sciences infirmières, les modalités proposées au travers des travaux de Freire (1998) se rapportent spécifiquement à un niveau de formation doctoral.

D'autres auteurs ont réfléchi l'utilisation de notions issues de la pensée de Freire pour concevoir certaines dimensions de la formation infirmière à visée clinique. Les travaux de Forneris et Peden-McAlpine (2006) proposent une utilisation de la notion de contextualisation des connaissances (Freire, 1970) pour soutenir le développement de stratégies pédagogiques dans la formation infirmière en vue d'une application clinique. La contextualisation, décrite dans les travaux de Freire (1970) comme la capacité à distinguer les éléments propres à un contexte donné et à ajuster ses actions en fonction de l'analyse d'une situation, vise un processus praxique et de réflexion critique plutôt qu'une application systématique de normes et de faits, tel que proposée par d'autres repères philosophiques, notamment ceux du courant post-positiviste. Waite et Brooks (2014) réfèrent pour leur part aux travaux de Freire (1970) pour réfléchir la notion de justice sociale dans le développement des compétences des étudiantes en sciences infirmières, sans toutefois fournir d'exemples quant au choix de contenus à mobiliser.

Rozendo, Santos Salas et Cameron (2017) proposent quant à eux une réflexion autour d'une interprétation de la notion de problématisation, telle que conçue par Freire (1970). La problématisation réfère ici à un processus de raisonnement, de questionnement et de réflexion critique sur l'ensemble des dimensions de la réalité, incluant les opportunités et les conséquences s'y rattachant (Rozendo et al., 2017). Ces auteurs soutiennent que le recours au processus de problématisation se développe, entre autres, par les approches pédagogiques expérimentées par l'étudiant et qu'à ce titre, les programmes de formation en sciences infirmières devraient fournir un espace permettant à l'étudiant d'en faire l'apprentissage (Rozendo et al., 2017). Mooney et Nolan (2006) adoptent une perspective similaire, suggérant que les notions issues des travaux de Freire (1970) pourraient permettre d'adopter des approches pédagogiques permettant une réflexion critique nécessaire à l'exercice de l'ensemble des compétences par les étudiants en sciences infirmières. Ces auteurs ne font toutefois pas de distinction entre les différents cycles d'études universitaires (Mooney et Nolan, 2006). Leyshon (2002), dans une visée similaire, propose une réflexion sur le développement du pouvoir d'agir de l'infirmière au cours de sa formation au travers de la notion de réflexion critique issue des travaux de Freire (1970). L'ensemble de ces auteurs soutiennent que la notion de réflexion critique devrait être centrale à la formation infirmière. Bien que contribuant à une réflexion nécessaire sur l'apport des travaux de Paulo Freire à la formation infirmière pour la pratique clinique, ces différents écrits n'offrent 
pas de repères concrets quant à de possibles implications pour la conceptualisation d'activités pédagogiques contextualisées ou de programmes de formation.

Ainsi, bien que ces quelques auteurs se soient penchés sur l'apport des travaux de Freire à la formation en sciences infirmières, aucun, à notre connaissance, n'a proposé d'activités pédagogiques concrètes s'adressant à de futures infirmières cliniciennes généralistes et s'appuyant sur ces repères conceptuels. De façon à potentialiser l'apport des travaux de Freire $(1970,1973,1998)$ à un apprentissage transformationnel en sciences infirmières, nous postulons qu'il est nécessaire d'illustrer de manière concrète une conceptualisation de leurs contributions. Ainsi, la prochaine rubrique propose la présentation d'une histoire de cas du développement d'un ensemble d'activités pédagogiques basé sur les repères issus des travaux de Freire (1970, 1973, 1998).

\section{Présentation du cours}

Les activités pédagogiques décrites dans le présent article ont été réfléchies dans le cadre du développement d'un cours s'inscrivant dans la formation de premier cycle en sciences infirmières au sein d'une université canadienne. Ce cours, qui se déroule dès les premières semaines de la formation universitaire des étudiants, vise une exploration des liens qui existent entre les notions de santé et d'environnement, un positionnement de la pratique infirmière future des étudiants intégrant ces notions et initie les étudiants à l'évaluation globale de la santé. Nous abordons la conceptualisation de ce cours avec l'idée qu'à ce stade de leur formation, les étudiants ont, pour la majorité, une vision et une compréhension observée ou racontée plutôt qu'expérientielle de la pratique infirmière.

Le cours est structuré au travers de trois types de modalités pédagogiques principalement actives, soit les présentations interactives en grand groupe, les ateliers et les activités d'intégration en petits groupes. Les présentations interactives en grand groupe constituent une formule plus classique, dans laquelle le professeur ou le conférencier invité présente un contenu pédagogique aux étudiants sous forme d'exposé. Les ateliers constituent une modalité pédagogique permettant aux étudiants, en petits groupes, d'explorer et d'approfondir certaines notions théoriques abordées dans un cours et laissent ainsi libre la mise en place de différentes activités d'apprentissage. Les activités d'intégration, quant à elles, bien qu'également réalisées en petits groupes, visent à se pencher sur une situation infirmière clinique présentée sous forme d'histoire de cas. Au sein du programme dans lequel s'inscrit le cours décrit, l'apprentissage par situations infirmières ou par problèmes (APP) est la méthode privilégiée pour favoriser l'apprentissage des étudiants.

Le cours se déroule sur une période de quatre semaines et équivaut à 4 crédits de cours ou 180 heures de travail pour l'étudiant. Cinq activités pédagogiques illustrées dans le présent article se dérouleront à différents moments dans la tenue du cours. La figure 1 illustre une représentation temporelle des différentes activités du cours développées à partir de la pensée de Freire. Les activités sont décrites et justifiées en s'appuyant sur les repères conceptuels décrits précédemment. 
Figure 1 - Représentation temporelle des activités pédagogiques

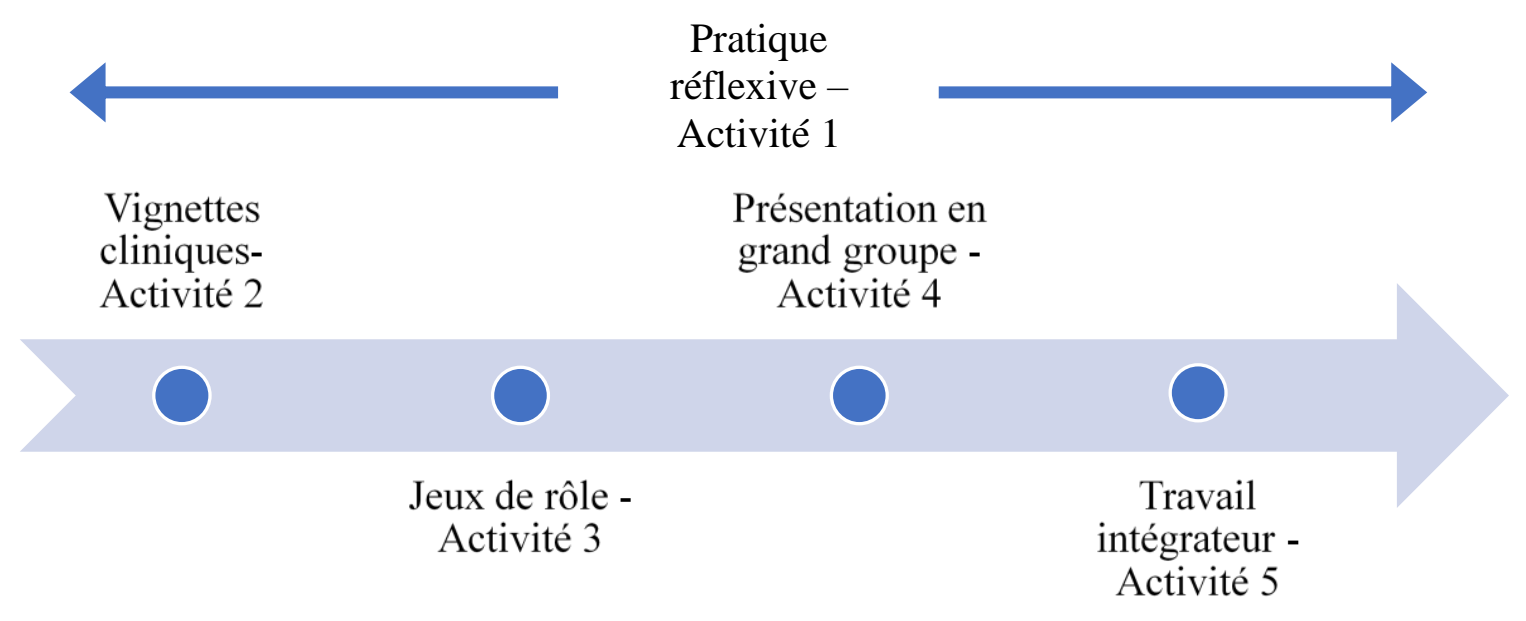

\section{Pratique réflexive}

La réflexion critique, effectuée par le biais d'une pratique réflexive sous forme écrite, fait partie intégrante de l'ensemble des modalités proposées dans ce cours, tant dans leur conception que lors d'évaluations certificatives. Il peut être déstabilisant, voire inconfortable pour l'apprenant d'être invité à répétition à exprimer ses réflexions quant à ses pensées et ses actions, de même qu'à certaines notions ou éléments du monde qui l'entoure (Freire, 1970). Pour cette raison, et considérant qu'il s'agit d'un cours qui s'inscrit au début de la formation des étudiants, nous introduisons la réflexion critique d'abord en expliquant les objectifs pédagogiques et en proposant d'amorcer un dialogue avec les étudiants sur cette activité. Cette introduction à la réflexion critique fournit des repères aux étudiants dans la réalisation d'un journal de bord réflexif qui s'étend sur l'ensemble du cours. L'étudiant est, d'une part, invité à réfléchir ses idées et ses questionnements quant à certaines notions abordées dans le cours, et d'autre part, à développer ses compétences à l'expression des éléments de sa pensée par l'apprentissage d'une structure de pensée réflexive. Ces notions incluent notamment les aprioris des étudiants quant au concept de santé ou quant aux liens entre la dimension technique du rôle de l'infirmière et une pratique salutogénique, orientée vers la qualité de vie et les actions génératrices de bien-être. La pratique réflexive, réalisée par l'entremise du journal de bord de manière individuelle, s'inscrit par ailleurs en continuité avec la réflexion critique mobilisée dans l'ensemble des modalités du cours et effectuée en interactions avec les pairs et l'enseignant. L'encadré 1 présente les liens conceptuels entre la pratique réflexive et la pensée de Freire. 
Encadré 1 - Pratique réflexive comme processus conscientisant

La réflexion critique, par un processus de conscientisation, est utilisée pour développer une plus grande conscience de soi ou d'une réalité donnée, ainsi que des rapports que nous entretenons avec cette réalité (Freire, 1998). En prenant comme point de départ la réalité, telle que construite par l'expérience et la pensée de l'étudiant, graduellement, celui-ci sera appelé à la comprendre, à s'y situer et à y agir de manière consciente. En effet, Freire $(1970,1973)$ soutient que le développement de la capacité critique de l'apprenant se développe par la conscience de ses pensées et de ses actions sur le monde social dans lequel il évolue.

\section{Vignettes cliniques}

$\mathrm{Au}$ travers de vignettes cliniques, les étudiants sont engagés dans un processus de résolution de problèmes infirmiers cliniques. Ces vignettes sont utilisées pour soutenir les discussions en petits groupes de travail et favoriser la contextualisation des apprentissages. Elles reposent sur un format de problématisation par le biais de discussions entre étudiants et avec l'enseignant pour illustrer la situation présentée et pour poser des actions infirmières et interprofessionnelles cohérentes. Considérant la vision idéalisée de la pratique infirmière souvent décrite dans les situations infirmières proposées, notre approche favorise une réintégration de la réalité clinique vécue par les infirmières, notamment des conditions de travail reflétant une charge de travail élevée, un manque de temps ou des collaborations interprofessionnelles difficiles. Cela permet à l'étudiant une construction de connaissances contextualisées en fonction de la réalité sociale, culturelle et politique, et ce, réfléchie de façon critique (Freire, 1998). Quoique parfois surprenante pour des infirmières en formation, cette réalité clinique complexe doit être reconnue dans la formation (Wolff, Pesut et Regan, 2010). Par cette introduction, les stratégies pour y développer sa pratique professionnelle reconnaissant la complexité de l'agir infirmier sont contextualisées au monde dans lequel les étudiants naviguent. L'encadré 2 présente les liens conceptuels entre les vignettes cliniques réalistes et la pensée de Freire.

Encadré 2 - Vignettes cliniques contextualisées

Freire (1970) conçoit la curiosité épistémique, soit la capacité de lier la théorie et la pratique par le questionnement des connaissances acquises ou développées, comme fondamental à l'apprentissage. Ces vignettes cliniques contextualisées visent à représenter une pratique infirmière politiquement, culturellement et socialement consciente dans laquelle les étudiants pourront réfléchir et appliquer les théories enseignées. Tel que conçu par Freire (1973), réfléchir la théorie sans la pratique n'est que verbalisme. Pour soutenir une conscientisation, le monde décrit ne doit pas être dissocié des réalités politiques ou culturelles, ainsi que des relations de pouvoir qu'elles constituent (Freire, 1973).

\section{Jeux de rôle}

$\mathrm{Au}$ travers des ateliers, nous proposons l'intégration de jeux de rôle, une activité pédagogique collaborative et discursive entre un petit groupe d'étudiants et un enseignant, afin de représenter et de vivre une pratique infirmière salutogénique. Pour ce faire, nous proposons d'utiliser les expériences cliniques vécues des enseignants afin de permettre une appropriation et une contextualisation qui tient compte de l'expérience clinique propre à chacun. Ces situations 
contextualisées permettront aux étudiants de réfléchir leurs évaluations et poser des actions conséquentes et conscientes. Considérant l'absence de pratique infirmière expérientielle chez ces étudiants si tôt dans leur formation, baser les activités sur le vécu des enseignants ouvre à une réinterprétation collaborative des situations de jeux de rôles. Cela pose une relation où tous, incluant l'enseignant, peuvent apprendre par la réflexion et la curiosité dans les situations présentées. L'encadré 3 présente les liens conceptuels entre les jeux de rôle et la pensée de Freire.

Encadré 3 - Jeux de rôle comme processus dialogique

Les jeux de rôles proposés sont en concordance avec la pédagogie fondée sur la problématisation, soutenue dans les écrits de Freire (1970). En effet, Freire (1970) conçoit que l'acte de conscientisation passe par le dialogue critique entre les étudiants et avec l'enseignement, tous dans un aplatissement des relations hiérarchiques convenues (Freire, 1970), c'est-à-dire que les relations sont plus horizontales que verticales ou hiérarchiques. L'enseignant n'effectue pas une transmission magistrale du savoir, mais crée les possibilités pour sa construction par les étudiants (Freire, 2006). Par la nécessité dialogique des situations proposées, par l'obligation d'action de tous les étudiants et par le rôle enseignant en soutien aux étudiants, cette méthode met en action une connaissance participative impliquant tous les acteurs (Freire, 2006). Le dialogue sur l'expérience de tous permet aux étudiants de s'approprier graduellement une réflexion sur les problèmes observés et vécus dans la réalité socioculturelle et y situer des pistes d'action. En écho à la pédagogie de l'autonomie (Freire, 2006), ce mode d'apprentissage est central au développement de savoirs contextualisés, agissants et conscients sur le monde.

\section{Présentation en grand groupe}

Tel que mentionné précédemment, le cours ci-décrit initie les étudiants à l'exploration du concept de santé dans la pratique infirmière, la santé vue comme une ressource positive soutenant une capacité d'action des personnes soignées. Cette conception de la santé, généralement en confrontation partielle avec la conception généralement décrite dans la sphère publique, pose le défi supplémentaire de son intégration à une pratique infirmière en bourgeonnement. Pour soutenir ce travail chez les étudiants, en plus d'introduire une réflexion sur leurs aprioris du concept de santé (voir activité de pratique réflexive), nous intégrons dans une présentation en grand groupe au cours de la session, des infirmières cliniciennes à titre de conférencières invitées. La compréhension clinique du rôle infirmière par les étudiants en première année au baccalauréat étant limitée, nous réfléchissons dans la venue des conférencières, une possibilité pour situer l'applicabilité d'une pratique salutogénique de l'infirmière. En proposant une illustration vécue de cette pratique, les conférencières fournissent une représentation de l'environnement de pratique et permettent d'engager les étudiants dans la réflexion et dans le questionnement sur leurs expériences antérieures et y concevoir les interrelations entre l'objet enseigné, la santé et la pratique infirmière vécue.

\section{Travail d'intégration}

Finalement, ce travail a un objectif intégrateur par la volonté de situer la future pratique infirmière des étudiants, au travers des concepts de santé et d'environnement qui ont été au centre du cours. L'étudiant y est invité à réfléchir les représentations possibles de la pratique salutogénique de l'infirmière dans un contexte de soins. Pour ce faire, les étudiants sont invités à 
décrire de manière factuelle une pratique infirmière vécue, soit comme patient, comme proche ou comme spectateur. S'ils n'ont pas vécu une expérience de santé auprès d'une infirmière, les étudiants sont appelés à concevoir une situation basée sur leur compréhension actuelle de la pratique infirmière. Cette expérience est utilisée comme point de départ à la construction d'une représentation d'une pratique infirmière clinique, expérientielle et théorique. Orientés par cette représentation, les étudiants sont invités à prendre le rôle de l'infirmière rencontrée; dans pareille situation, comment auraient-ils situé la santé dans leur pratique infirmière? Quelles évaluations auraient été effectuées et pourquoi? Quelles sont l'analyse et l'interprétation de cette situation? Et finalement, quelles sont les pistes d'action? Ce travail, orienté autour d'une démarche de soins infirmiers, permet à l'étudiant de poser un regard critique sur les liens qui existent entre sa propre santé physique ou mentale et son environnement physique et social, en plus d'y situer la pratique infirmière et son action. Ce travail final réintroduit l'expérience étudiante avec les établissements de soins comme toile de fond de la réflexion sur la future pratique. La nature praxéologique de ce travail initie un processus de réflexion sur la santé de l'étudiant, comme acteur social et professionnel. Par la mise en action du concept de conscientisation (Freire, 1970, 1973), l'étudiant est engagé dans une réflexion sur la réalité vécue, et est amené à une prise de conscience critique des rapports sociaux qu'il a de façon concomitante comme personne soignée et comme professionnel.

\section{Discussion}

Les activités pédagogiques abordées dans la section précédente et contextualisées à un cours permettent d'illustrer une opérationnalisation possible des concepts issus de la pensée de Freire (1973). Une conception de l'apprentissage à visée transformationnelle, soutenue par les notions d'interactions, de contextualisation et de réflexion critique, permet à l'apprenant d'acquérir les outils individuels ou collectifs qui soutiendront tant une conscientisation de la réalité socioculturelle qui structure sa vie, que de sa capacité à transformer cette réalité (Freire, 1973). Au travers de la conscientisation de l'être-sujet, Freire (1970) nous offre un référent conceptuel phare pour répondre aux critiques formulées par Frenk et al. (2010) et Benner et al. (2010).

Bien que dans le présent article, ces notions aient été utilisées pour l'élaboration d'activités pédagogiques se rapportant à un cours spécifique d'un programme de formation infirmière qui offre une pédagogie active, nous postulons qu'elles peuvent contribuer plus largement à répondre à certains enjeux de la formation infirmière soulevés dans les travaux de Benner et al. (2010).

Une des lacunes soulevées par Benner et al. (2010) se rapporte au trop peu de soutien qu'offrent les cours à la poursuite de l'apprentissage clinique des étudiants. Cette lacune se manifeste en pratique par un manque de compétences des infirmières à utiliser des ressources diverses pour répondre à des interrogations cliniques et ainsi soutenir l'actualisation des connaissances et des aptitudes au cours de leur carrière et en fonction des différents milieux dans lesquels elles évoluent (Benner et al., 2010). Benner et al. (2010) soutiennent que le recours à une pédagogie inquisitive, soit une pédagogie dans laquelle la curiosité de l'étudiant est centrale et sert de levier à ses apprentissages, pourrait être particulièrement favorable à la pratique, notamment en réponse aux exigences grandissantes du système de santé. Ce type de pédagogie place l'étudiant dans une position où il doit développer des compétences et poursuivre ses apprentissages suivant la fin de son parcours académique. Ce positionnement de Benner et al. 
(2010) envers la pertinence d'une telle pédagogie rejoint les éléments centraux de la conception de l'éducation proposée par Freire (1970).

Les notions d'interactions, de contextualisation et de réflexion critique utilisées dans l'opérationnalisation de nos activités s'inscrivent dans une pédagogie inquisitive. Freire (1970) conçoit que le développement des outils nécessaires à un agir conscient dans le monde requiert de l'étudiant un rôle d'apprenant actif. La situation d'apprentissage, qui se déroule autour d'un objet connaissable, consiste en un processus qui permet d'outiller l'apprenant à la poursuite d'autres apprentissages plutôt qu'à une finalité (Freire, 1970). Selon cette conception, les connaissances développées à partir d'un objet connaissable varient en fonction des acteurs qui interagissent avec cet objet. Cette réflexion requiert un engagement de l'étudiant, sans quoi le développement de connaissances est impossible. Freire (1970) conçoit ainsi la connaissance comme émergeant d'un processus dynamique d'interactions impliquant une création et une réinvention des savoirs. La mise en place de cette dynamique est essentielle pour maintenir l'étudiant dans une position d'apprenant actif et pour susciter sa conscientisation. Cette dynamique risquant toutefois de déstabiliser l'étudiant, Freire (1970) conçoit que ses effets bénéfiques sur ses apprentissages apparaissent de façon évolutive, soit en y exposant de plus en plus l'étudiant, durant l'ensemble de son parcours académique.

En lien avec les limites actuelles relevées par Benner et al. (2010), nous concevons que le propos de Freire (1970) sur la pédagogie inquisitive offre deux principales avenues pour réfléchir la formation infirmière. D'abord, l'opérationnalisation des notions d'interactions, de contextualisation et de réflexion critique pourrait être effectuée à l'ensemble d'un programme de formation infirmière. Une orientation explicite et commune de l'ensemble des activités pédagogiques, plutôt que de guider de façon normative le contenu présenté aux étudiants (Freire, 1998), pourrait permettre de rendre cohérente et plus uniforme la façon d'aborder ce contenu au travers de modalités pédagogiques (Feller, 2018). Cette cohérence, pour Freire (1970), est essentielle afin de permettre aux étudiants d'apprivoiser cette pédagogie inquisitive.

Ensuite, nous postulons que les repères conceptuels issus de la pensée de Freire (1970, 1973) pourraient être utilisés pour réfléchir la place des programmes de formation en sciences infirmières dans la réponse à certains enjeux actuels de la pratique. Le large éventail de compétences attendues des infirmières, la diversité des milieux de travail ainsi que la complexité de la pratique infirmière, le développement de la technologie et la diversité des milieux de travail, (Benner et al., 2010; Duncan, Rodney et Thorne, 2014; Duncan, Thorne, Van NesteKenny et Tate, 2012; Thorne, 2006) exigent une formation qui va au-delà de la transmission de connaissances. En relevant le besoin de mieux soutenir la capacité des étudiants à poursuivre leurs apprentissages et à développer leurs ressources, Benner et al. (2010) suggèrent que les programmes de formation en sciences infirmières devraient avoir comme objectif d'outiller les étudiants, plutôt que de transmettre des connaissances les rendant aptes à pratiquer dans divers domaines. Bien que Benner et al. (2010), dans leurs travaux sur la formation infirmière, n'établissent pas de liens explicites avec la pensée de Freire, nous concevons, tel qu'illustré, que plusieurs concepts réfléchis par ce philosophe pourraient alimenter conceptuellement la poursuite des réflexions liées aux enjeux soulevés par ces auteurs quant à l'orientation et la structure de la formation universitaire en sciences infirmières. 


\section{Implications pour la formation en sciences infirmières}

Nous concevons diverses implications inhérentes à l'adoption d'une pédagogie inquisitive s'inscrivant dans une perspective freirienne pour réfléchir la formation en sciences infirmières. Une implication centrale concerne la façon dont les différents acteurs impliqués dans l'apprentissage et la pratique conçoivent la finalité de la formation.

De la même façon que Pepin et al. (2017), nous concevons qu'un arrimage entre les milieux académique et clinique est nécessaire de façon à assurer une cohérence entre la finalité recherchée par la formation infirmière en milieu académique et celle attendue dans les milieux de pratique. S'inspirer de la pensée de Freire comme repère conceptuel à la formation infirmière oblige à reconnaître l'interdépendance qui doit exister entre les milieux académique et clinique (Frenk et al., 2010). Selon cette perspective, les apprentissages d'une pratique infirmière ne se rapporteraient pas uniquement aux milieux académiques. L'apprentissage étant conçu comme un processus évolutif plutôt qu'une finalité (Freire, 1970), il nécessite une implication des milieux cliniques dans la poursuite des apprentissages des infirmières au terme de la formation académique.

Dans un autre ordre d'idées, s'inspirer d'une pédagogie inquisitive freirienne implique une réflexion sur la perception des enseignants quant à leur contribution dans la préparation des étudiants à une pratique professionnelle. La pédagogie telle que conçue par Freire (1970), en définissant l'objet connaissable comme dépendant d'un partage entre l'enseignant et l'apprenant plutôt que transmis par l'enseignant, requiert inévitablement une remise en question du rôle de ce dernier. La cohérence dans l'orientation d'un programme débute par une orientation claire au sein des membres enseignants (Benner et al., 2010). Si les formateurs des programmes universitaires en sciences infirmières ont l'obligation de faciliter la navigation des étudiants dans le pluralisme théorique afin de permettre leur rôle professionnel dans la complexité renouvelée, une réflexion s'impose quant à une approche pédagogique explicite et commune qui sous-tend cet accompagnement.

Finalement, les étudiants, étant conçus comme des apprenants actifs et dont la curiosité épistémique est essentielle au développement des apprentissages, nous concevons que les implications d'une pédagogie inspirée des travaux de Freire concernent également ces acteurs. Une perspective freirienne conçoit l'apprenant comme étant non seulement actif dans ses apprentissages, mais comme étant responsable des connaissances qu'il développe (Freire, 1970) en interactions avec les membres enseignants et ses pairs. Cette approche pédagogique requiert inévitablement un engagement de l'étudiant, non seulement dans son propre parcours académique, mais comme acteur dans les apprentissages des autres (Freire, 1970). Dans un objectif de développement de la formation autour d'une pensée freirienne, une réflexion s'impose sur la préparation des étudiants à cette pédagogie active et dialogique.

Si la proposition présentée dans cet article ne se rapporte qu'à un cours spécifique, nous postulons, à partir de la conception de l'éducation de Freire (1970), qu'il est nécessaire d'y réfléchir plus largement pour concevoir un programme de formation universitaire en sciences infirmières. Nous concevons particulièrement comme porteuse l'amorce d'une réflexion quant au rapprochement à faire entre les lacunes évoquées par Benner et al. (2010) et la pertinence de développer une pédagogie inquisitive basée sur l'apprentissage transformationnel, illustrée ici par la pensée de Freire (1970) dans la formation infirmière. 


\section{Conclusion}

Dans ce texte, la mise en action de la pédagogie freirienne (1970) offre une représentation unique de l'influence des repères conceptuels dans la création d'activités pédagogiques en formation infirmière. De par cette influence, il est essentiel en tant que formateurs, de mettre en lumière et de questionner les repères conceptuels ou théoriques qui orientent la formation. Donohue-Porter, Forbes et White (2011) mentionnent que la formation infirmière, lorsqu'elle est soutenue conceptuellement, permet une cohésion entre les cours du programme, influence les choix pédagogiques du corps enseignant et expose les étudiants à la pluralité des modes de développement de savoirs. Cependant, ces auteurs mentionnent que le recours à des repères conceptuels est insuffisant au sein des programmes de formation en sciences infirmières (Donohue-Porter et al., 2011). Cela entraîne une formation parcellaire, morcelée et intégrant peu le développement professionnel et identitaire. Dans l'objectif de soutenir une formation reconnaissant et intégrant la complexité des pratiques actuelles (Benner et al., 2010), l'utilisation de repères théoriques dans la conception de cours et de programmes doit être systématisée (Donohue-Porter et al., 2011). Une approche en formation basée sur de tels repères pourrait permettre non seulement de répondre à certains enjeux entourant la formation et la pratique infirmières, mais contribuerait également à la construction de l'identité professionnelle de l'infirmière. Cette identité implique une conscientisation et une reconnaissance de ses actions en tant qu'acteur au sein d'un système de santé, qui demeure un enjeu central à l'évolution de la pratique infirmière (Duncan et al., 2014). Qu'ils soient singuliers ou pluriels, ces repères sont centraux au processus de transformation radicale de la formation infirmière de premier cycle universitaire qui mène au déploiement d'une pratique infirmière évolutive et renouvelée. 


\section{Références}

Benner, P., Sutphen, M., Leonard, V., et Day, L. (2010). Educating nurses: A call for radical transformation. San Francisco, CA: Jossey-Bass.

Bond Butts, J., et Saucier Lundy, K. (2003). Teaching Philosophy of Science in Nursing Doctoral Education. Journal of Nursing Scholarship, 35(1), 87-91. https://doi.org/10.1111/j.1547-5069.2003.00087.x

Chinn, P. L., et Kramer, M. K. (2015). Knowledge Development in Nursing. Theory and Process. ( $9^{\mathrm{e}}$ éd.). St. Louis, MI: Mosby, Elsevier.

Donohue-Porter, P., Forbes, M. O., et White, J. H. (2011). Nursing Theory in Curricula Today: Challenges for Faculty at all Levels of Education. International Journal of Nursing Education Scholarship, 8. https://doi.org/10.2202/1548-923x.2225

Duncan, S., Rodney, P. A. et Thorne, S. (2014). Forging a strong nursing future: insights from the Canadian context. Journal of Research in Nursing, 19(7-8), 621-633. https://doi.org/10.1177/1744987114559063

Duncan, S. M., Thorne, S., Van Neste-Kenny, J., et Tate, B. (2012). Policy analysis and advocacy in nursing education: The Nursing Education Council of British Columbia framework. Nurse Education Today, 32(4), 432-437. https://doi.org/10.1016/j.nedt.2011.03.009

Feller, F. (2018). Transforming Nursing Education: A Call for a Conceptual Approach. Nurs Educ Perspect, 39(2), 105-106. https://doi.org/10.1097/01.nep.0000000000000187

Flexner, A. (1910). Medical education in the United States and Canada: a report to the Carnegie Foundation for the Advancement of Teaching. New York, NY: The Carnegie Foundation for the Advancement in Teaching.

Forneris, S. G., et Peden-McAlpine, C. J. (2006). Contextual Learning: A Reflective Learning Intervention for Nursing Education. International Journal of Nursing Education Scholarship, 3. https://doi.org/10.2202/1548-923x.1254

Freire, P. (1970). Pedagogy of the oppressed. (Traduit par M. B. Ramos). New York, NY: Continuum International Publishing Group.

Freire, P. (1973). Education for Critical Consciousness. New York, NY: Continuum.

Freire, P. (1998). Pedagogy of Freedom. Ethics, Democracy and Civic Courage. (Traduit par P. Clarke). Lanhan, MD: Rowan \& Littlefield Publishers, Inc.

Freire, P. (2006). Pégagogie de l'autonomie. Toulouse, France: Eres.

Frenk, J., Chen, L., Bhutta, Z. A., Cohen, J., Crisp, N., Evans, T., ... Zurayk, H. (2010). Health professionals for a new century: transforming education to strengthen health systems in an interdependent world. Lancet, 376(9756), 1923-1958. https://doi.org/10.1016/s0140$\underline{6736(10) 61854-5}$

Goudreau, J., Pepin, J., Dubois, S., Boyer, L., Larue, C., et Legault, A. (2009). A second generation of the competency-based approach to nursing education. International Journal of Nursing Education Scholarship, 6, Article 15. https://doi.org/10.2202/1548-923x.1685 
Ironside, P. M. (2001). Creating a research base for nursing education: an interpretive review of conventional, critical, feminist, postmodern, and phenomenologic pedagogies. ANS Advances in Nursing Science, 23(3), 72-87. https://doi.org/10.1097/00012272200103000-00007

Leyshon, S. (2002). Empowering practitioners: an unrealistic expectation of nurse education? Journal of Advanced Nursing, 40(4), 466-474. https://doi.org/10.1046/j.13652648.2002.02395.x

McAllister, M. (2011). STAR: A Transformative Learning Framework for Nurse Educators. Journal of Transformative Education, 9(1), 42-58. https://doi.org/10.1177/1541344611426010

Mooney, M., et Nolan, L. (2006). A critique of Freire's perspective on critical social theory in nursing education. Nurse Education Today, 26(3), 240-244. https://doi.org/10.1016/j.nedt.2005.10.004

Pepin, J., Goudreau, J., Lavoie, P., Bélisle, M., Blanchet Garneau, A., Boyer, L., ... Lechasseur, K. (2017). A nursing education research framework for transformative learning and interdependence of academia and practice. Nurse Education Today, 52, 50-52. https://doi.org/10.1016/j.nedt.2017.02.001

Rich, K. L., et Nugent, K. E. (2010). A United States perspective on the challenges in nursing education. Nurse Educ Today, 30(3), 228-232. https://doi.org/10.1016/j.nedt.2009.10.015

Rosenau, P., Watson, L., Vye-Rogers, L. et Dobbs, M. (2015). Educating for complexity In nursing practice: A baccalaureate curriculum innovation. Quality Advancement in Nursing Education - Avancées en formation infirmière, 1(3). https://doi.org/10.17483/2368-6669.1039

Rozendo, C. A., Santos Salas, A., et Cameron, B. (2017). Problematizing in nursing education: Freire's contribution to transformative practice. Nurse Education Today, 51, 120-123. https://doi.org/10.1016/j.nedt.2016.08.009

Thorne, S. E. (2006). Nursing education: Key issues for the 21st century. Nurse Education in Practice, 6(6), 306-313. https://doi.org/10.1016/j.nepr.2006.07.006

Waite, R., et Brooks, S. (2014). Cultivating social justice learning \& leadership skills: a timely endeavor for undergraduate student nurses. Nurse Educ Today, 34(6), 890-893. https://doi.org/10.1016/j.nedt.2014.02.009

Wolff, A. C., Pesut, B. et Regan, S. (2010). New graduate nurse practice readiness: perspectives on the context shaping our understanding and expectations. Nurse Educ Today, 30(2), 187-191. https://doi.org/10.1016/j.nedt.2009.07.011 